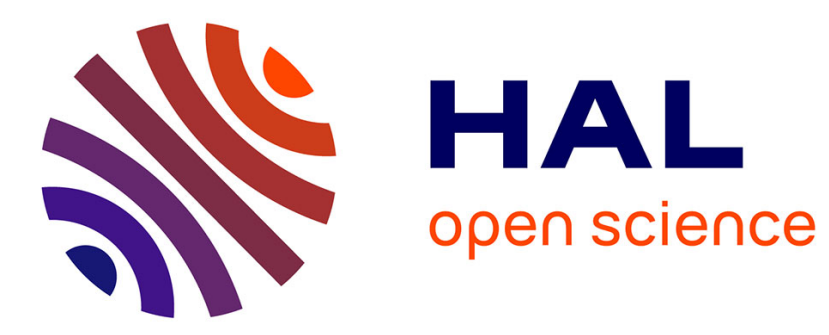

\title{
Paramagnétisme des ferrites de fer, de cobalt, de nickel
} Muriel Fallot, P. Maroni

\section{To cite this version:}

Muriel Fallot, P. Maroni. Paramagnétisme des ferrites de fer, de cobalt, de nickel. J. Phys. Radium, 1951, 12 (3), pp.256-257. 10.1051/jphysrad:01951001203025600 . jpa-00234378

\section{HAL Id: jpa-00234378 https://hal.science/jpa-00234378}

Submitted on 1 Jan 1951

HAL is a multi-disciplinary open access archive for the deposit and dissemination of scientific research documents, whether they are published or not. The documents may come from teaching and research institutions in France or abroad, or from public or private research centers.
L'archive ouverte pluridisciplinaire HAL, est destinée au dépôt et à la diffusion de documents scientifiques de niveau recherche, publiés ou non, émanant des établissements d'enseignement et de recherche français ou étrangers, des laboratoires publics ou privés. 


\title{
PARAMAGNÉTISME DES FERRITES DE FER, DE GOBALT, DE NIGKEL
}

\author{
Par M. FALLOT et P. MARONI.
}

\begin{abstract}
Sommaire. - Les auteurs étudient Je paramagnétisme des ferrites de fer, cobalt, nickel, avec une précision accrue, et dans un intervalle compris entre je point de Curie et $1200^{\circ} \mathrm{C}$. Les résultats expérimentaux sont représentés correctement par la loi hyperbolique de Néel. Les valeurs trouvées pour les constantes de Curie sont plus élevées que celles prévues par l'hypothèse de la constance des coefficients de champ moléculaire.
\end{abstract}

Les connaissances que l'on possède jusqu'à présent [1] sur les ferrites de fer et de nickel, ne permettent pas d'obtenir une loi précise de la variation thermique de leur susceptibilité magnétique : les repères de températures ne sont pas corrects dans les expériences les plus anciennes et d'une manière générale l'intervalle de température exploré est insuffisant; sur le ferrite de cobalt, on ne possède aucun résultat.

Nos expériences sur ces substances ont été faites dans le domaine de températures compris entre leurs points de Curie et $\mathrm{I}_{200^{\circ}} \mathrm{C}$. La méthode employée, basée sur l'attraction dans un champ non uniforme, utilise une balance de translation de Foëx et Forrer [2], étalonnée par le pyrophosphate de manganèse et dont le four ainsi que le dispositif de mesure des températures ont été précédemment décrits [3].

Les substances étudiées ont été une magnétite naturelle et des ferrites de nickel ou de cobalt préparés au Laboratoire par réduction des oxalates ou des formiates. En ce qui concerne le premier de ces corps, on a pu constater qu'une oxydation, lente entre 900 et ${ }^{1000^{\circ}}$, rapide au-dessus de ${ }^{\circ} 000^{\circ}$, est cause d'une incertitude sur les mesures effectuées dans l'air; au contraire, les courbes obtenues en opérant sur une magnétite scellée sous vide dans un tube de quartz sont parfaitement réversibles. Les ferrites de nickel et cobalt, après recuit à $\mathrm{r} 200^{\circ}$, sont très stables et peuvent être chauffés à l'air. Les mesures ont été reproductibles à $\mathrm{I} / \mathrm{I}_{\mathrm{O}} \mathrm{oo}^{\mathrm{e}}$ près avec les échantillons utilisés (ferrites en poudre ou agglomérés).

Le graphique ci-contre donne en traits pleins les résultats expérimentaux et montre la variation thermique des susceptibilités, celles-ci étant rapportées aux molécules $\mathrm{Fe}_{3} \mathrm{O}_{4} ; \mathrm{Fe}_{2} \mathrm{O}_{3} \mathrm{CoO} ; \mathrm{Fe}_{2} \mathrm{O}_{3} \mathrm{NiO}$.

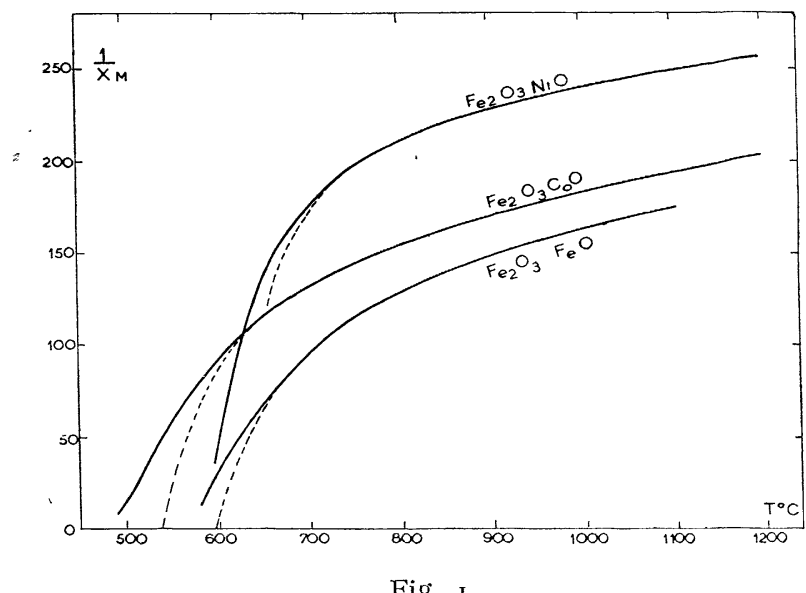

Nous avons essayé d'interpréter l'allure de ces courbes en leur assignant une loi hyperbolique ainsi que l'a proposé L. Néel [4].

$$
\frac{\mathrm{I}}{\bar{\gamma}_{\mathrm{II}}}=\frac{T}{C}+\frac{\mathrm{I}}{\%_{0}}-\frac{\sigma}{T-\theta}
$$

Les quatre constantes $C, \%, \sigma, 0$ peuvent être déterminées à partir des données expérimentales par une méthode graphique d'approximations successives; ce traitement conduit aux résultats suivants :

Tableav.

\begin{tabular}{|c|c|c|c|c|c|c|c|c|c|}
\hline 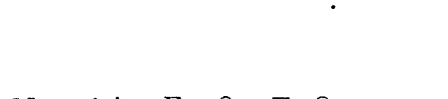 & $C^{\prime}$ & $C$. & $\frac{1}{\%}$ & $\sigma \cdot$ & $\begin{array}{c}\theta \\
(\circ \mathrm{K}) .\end{array}$ & $\begin{array}{c}\theta_{p} \\
(\circ \mathrm{K}) .\end{array}$ & $\begin{array}{c}0_{f} \\
\left({ }^{\circ} \mathrm{K}\right)\end{array}$ & $\begin{array}{c}\theta_{p}-0_{f} \\
(\circ) .\end{array}$ & $\begin{array}{l}\text { Intervalle } \\
\text { de validite } \\
\quad\left({ }^{\circ} \mathrm{C}\right) .\end{array}$ \\
\hline Magnétite $\mathrm{Fe}_{2} \mathrm{O}_{3}, \mathrm{FeO}$. & 11,9 & 14,2 & 103,2 & Iวั 100 & 777 & 869 & 847 & +22 & $700-1100$ \\
\hline Ferrite Co $\ldots \ldots \ldots \ldots \ldots \ldots$ & I I, 8 & I 3,9 & I I I, 9 & I I ǰjo & 744 & 812 & 769 & +43 & ZOO-I 200 \\
\hline Ferrite $\mathrm{xi} \ldots \ldots \ldots \ldots \ldots \ldots$ & Io, $\mathrm{C}$ & 19,5 & I $9 \mathrm{I}, 8$ & 7750 & 863 & 896 & 870 & +26 & $800-1200$ \\
\hline
\end{tabular}


On constate qu'aux températures assez élevées les valeurs indiquées pour les constantes permettent de calculer les susceptibilités à partir de la formule de Néel avec une exactitude de l'ordre de $\mathrm{I} / 2$ ooo c'est-à-dire avec une approximation supérieure aux incertitudes expérimentales. Tandis que dans un intervalle de $\mathrm{I}_{50^{\circ}}$ environ au-dessus du point de Curie, par suite des fluctuations du champ moléculaire, la loi de Néel conduit aux courbes tracées en traits ponctués.

Les points de Curie paramagnétiques $\theta_{p}$ sont supérieurs de 20 à $40^{\circ}$ aux points de Curie ferro- magnétiques 0 f déterminés par R. Pauthenet [5], en accord avec les résultats antérieurs.

Malgré la précision des mesures, les constantes $C$ de Curie-Néel, représentées par la pente de l'asymptote à l'hyperbole (1), ne peuvent être déterminées qu'à deux ou trois pour ioo près. Elles sont nettement supérieures aux constantes de Curie $C^{\prime}$ calculées à partir des moments, en tenant compte du moment orbital, et correspondant à tous les ions $\mathrm{Fe}, \mathrm{Co}, \mathrm{Ni}$. Les écarts, tous dans le même sens, peuvent s'expliquer par la variation thermique des coefficients de champ moléculaire.

\section{BIBLIOGRAPHIE.}

[1] Curie P. - Ann. Chim. Phys., i 895, 5, 289. - Kopp W. - Thèse, Zurich, r919. - Serres A. - Ann. Phys., I $932, \mathbf{1 7}, 5$.

[2] Foex G. et Forrer R. - J. Phys. Rad., 1926, 7, 180.
[3] Fallot M. - J. Phys. Rad., i94年, 5, i 53.

[4] Néel L. - Ann. Physique, r 948, 3, 137.

[5] Pauthenet R. - C. R. Acad. Sci., 1950, 230, 1842. 